\title{
O padrão da rotura do supraespinal afeta os resultados do reparo artroscópico?*
}

\section{Does the Supraspinatus Tear Pattern Affect the Results of the Arthroscopic Repair?}

\author{
Eduardo Angeli Malavolta ${ }^{1}$ Fernando Brandão Andrade-Silva ${ }^{1}$ André Lange Canhos ${ }^{1}$ \\ Jorge Henrique Assunção ${ }^{10}$ Mauro Emilio Conforto Gracitelli ${ }^{1}$ Arnaldo Amado Ferreira Neto ${ }^{1}$
}

1 Grupo de Ombro e Cotovelo, Instituto de Ortopedia e Traumatologia, Hospital das Clínicas, Faculdade de Medicina, Universidade de São Paulo (HCFMUSP), São Paulo, SP, Brasil
Endereço para correspondência Jorge Henrique Assunção, PhD, Rua Dr. Ovídio Pires de Campos, 333, $3^{\circ}$ andar, São Paulo, SP, 05403-010, Brasil (e-mail: drjorgeassuncao@gmail.com).

Rev Bras Ortop 2020;55(6):742-747.

\section{Resumo \\ Palavras-chave \\ - manguito rotador \\ - artroscopia \\ - amplitude de movimento articular \\ Objetivo Avaliar a influência do padrão da rotura do supraespinal nas avaliações funcionais pré e pós-operatória. \\ Métodos Estudo de coorte retrospectivo, comparando pacientes com rotura do supraespinal em crescente versus em $\mathrm{L}$ ou $U$. Incluímos pacientes submetidos ao reparo artroscópico completo do supraespinal. Não incluímos pacientes com reparo dos tendões do subescapular ou infraespinal, aqueles submetidos a cirurgia aberta, ou aqueles nos quais foi obtido apenas o reparo parcial. As escalas clínicas utilizadas foram The American Shoulder and Elbow Surgeons Standardized Shoulder Assessment (ASES) e Modified-University of California at Los Angeles Shoulder Rating Scale (UCLA), aplicadas uma semana antes e 24 meses após o procedimento. \\ Resultados Analisamos 167 ombros (de 163 pacientes). No pré-operatório, a escala da ASES demonstrou ser significativamente superior no padrão em crescente $(43,5 \pm 17,6$ versus $37,7 \pm 13,8 ; p=0,034)$. A escala da UCLA teve o mesmo padrão $(15,2 \pm 4,6$ versus $13,5 \pm 3,6 ; p=0,028)$. No pós-operatório, entretanto, não ocorreu diferença significativa. De acordo com a escala da ASES, roturas em crescente tiveram $83,7 \pm 18,7$ pontos, e as roturas em $\mathrm{L}$ ou $\mathrm{U}, 82,9 \pm 20,1(p=0,887)$. Respectivamente, os valores foram de $30,9 \pm 4,9$ e $30,5 \pm 5,6(p=0,773)$ pela escala da UCLA. \\ Conclusão As roturas em crescente e em $L$ ou $U$ do supraespinal apresentam resultados funcionais pós-operatórios semelhantes. No pré-operatório, os resultados funcionais são superiores nas roturas em crescente.}

Abstract

Objective To evaluate the influence of the supraspinal tear pattern on the pre- and postoperative functional evaluations.
Trabalho desenvolvido no Grupo de Ombro e Cotovelo, Instituto de Ortopedia e Traumatologia, Hospital das Clínicas, Faculdade de Medicina, Universidade de São Paulo (HCFMUSP), São Paulo, SP, Brasil. recebido

13 de Dezembro de 2018 aceito 31 de Julho de 2019
DOI https://doi.org/ 10.1055/s-0039-1698803. ISSN 0102-3616.
Copyright $\odot 2020$ by Sociedade Brasileira License terms de Ortopedia e Traumatologia. Published by Thieme Revinter Publicações Ltda, Rio de Janeiro, Brazil 


\section{Keywords \\ - rotator cuff \\ - arthroscopy \\ - articular range of motion}

Methods A retrospective cohort study comparing patients with supraspinatus crescent-shaped tears versus L- or U-shaped tears. We included patients undergoing complete supraspinatus arthroscopic repair. We did not include patients with subscapularis or infraspinatus repair, those submitted to open surgery, or those in whom only partial repair was obtained. The clinical scales used were the American Shoulder and Elbow Surgeons Standardized Shoulder Assessment (ASES) and the ModifiedUniversity of California at Los Angeles Shoulder Rating Scale (UCLA), which were applied 1 week before and 24 months after the procedure.

Results We analyzed 167 shoulders (from 163 patients). In the preoperative period, the ASES scale was significantly higher for the crescent-shaped pattern $(43.5 \pm 17.6$ versus $37.7 \pm 13.8 ; p=0.034)$. The UCLA scale followed the same pattern $(15.2 \pm 4.6$ versus $13.5 \pm 3.6 ; p=0.028)$. In the postoperative period, however, there was no significant difference. According to the ASES scale, crescent-shaped tears scored $83.7 \pm 18.7$ points, and L- or U-shaped tears scored $82.9 \pm 20.1(p=0.887)$. The values were $30.9 \pm 4.9$ and $30.5 \pm 5.6(p=0.773)$ respectively, by the UCLA scale.

Conclusion Crescent-shaped and L- or U-shaped Supraspinatus tears have similar postoperative functional results. In the preoperative period, the functional results are superior in crescent-shaped tears.

\section{Introdução}

A rotura do manguito rotador acomete $20 \%$ da população geral e até $50 \%$ dos pacientes acima de 80 anos. ${ }^{1}$ A melhora clínica após o procedimento cirúrgico ocorre na maioria dos pacientes, ${ }^{2-4}$ mas rerroturas ocorrem em $27 \%$ dos casos. ${ }^{5}$

A avaliação de fatores preditivos é importante para definir pacientes de risco para resultados insatisfatórios após o reparo do manguito rotador. Existem alguns estudos que avaliam fatores que aumentam o risco de falha de cicatrização ${ }^{6-12} \mathrm{e}$ de resultados clínicos desfavoráveis. ${ }^{13-16}$ Já foram descritos como fatores de risco para piores resultados clínicos: pacientes mais idosos; ${ }^{14,15}$ sexo feminino; ${ }^{15}$ pior função pré-operatória; cirurgia prévia e problemas trabalhistas; $;{ }^{16}$ tabagismo; ${ }^{17}$ grau de degeneração gordurosa; e dimensão da rotura. ${ }^{18}$

As roturas do manguito rotador têm padrões estruturais distintos, classicamente descritos como crescente (C), L, e U. ${ }^{19}$ Até o momento, poucos estudos avaliaram a influência do padrão da rotura nos resultados clínicos pós-cirúrgi$\cos ,{ }^{20,21}$ sem evidência de diferença entre os grupos. Estes estudos incluíram em sua casuística roturas do infraespinal $^{20,21}$ e do subescapular, ${ }^{20}$ o que, embora aumente a validade externa dos resultados, aumenta os fatores de confusão. $O$ objetivo deste estudo é analisar a influência do padrão da rotura do supraespinal na avaliação funcional pré e pós-operatória.

\section{Métodos}

\section{Desenho}

Estudo de coorte retrospectivo, comparando a avaliação funcional pré e pós-operatória entre dois grupos de pacientes, de acordo com o padrão da rotura do supraespinal: C versus $\mathrm{L}$ ou $\mathrm{U}$.

\section{Local e datas}

Foram analisados pacientes submetidos a reparo artroscópico completo do supraespinal com procedimentos realizados entre novembro de 2012 e novembro de 2016, por um dos médicos do Grupo de Ombro e Cotovelo de nossa instituição.

\section{Procedimento cirúrgico e reabilitação}

As cirurgias foram realizadas por artroscopia, sob anestesia geral e bloqueio interescalênico. Os pacientes foram posicionados em cadeira de praia ou decúbito lateral, de acordo com a preferência do cirurgião. Bursectomia, acromioplastia e ressecção distal da clavícula foram realizadas conforme necessário. O manguito rotador foi reparado após o desbridamento do tubérculo maior com técnica de fileira simples, utilizando âncoras duplamente carregadas. 0 tendão da cabeça longa do bíceps foi abordado quando apresentava subluxação ou luxação, lesões parciais superiores a $50 \%$, ou na existência de lesões do lábio superior dos tipos 2,3 e 4 . O procedimento realizado era a tenotomia, nos pacientes com idade maior ou igual a 60 anos, ou a tenodese, nos pacientes mais novos. A tenodese, quando indicada, era realizada ou com um dos fios da âncora mais anterior, ou com uma âncora especificamente para esse fim. Antes de se iniciar o reparo do manguito rotador, a lesão era mensurada com o auxílio de uma probe milimetrada, e o padrão era avaliado conforme a redutibilidade dela ao leito ósseo. $O$ número de âncoras e a necessidade de pontos tendão-tendão foi decidido no decorrer do ato cirúrgico.

Após a cirurgia, os pacientes permaneceram por 6 semanas imobilizados com tipoia Velpeau. Movimentos com os dedos, punho e cotovelo foram incentivados desde o primeiro dia. Arco de movimento passivo do ombro foi iniciado com quatro semanas, e movimentos ativos, após a retirada da tipoia. Fortalecimento foi realizado após os três meses, e liberação completa para as atividades laborais e esportivas, aos seis meses. 


\section{Ressonância magnética}

Todos os pacientes realizaram ressonância magnética (RM) previamente ao procedimento cirúrgico, num aparelho de 1,5-T (HDxt, GE Medical Systems, Milwaukee, WI, EUA) e bobina para ombro, sem uso de contraste intra-articular ou intravenoso.

\section{Participantes (critérios de eligibilidade)}

Foram incluídos pacientes submetidos a cirurgia artroscópica para tratamento de roturas isoladas do supraespinal, com reparo completo. Os pacientes ainda precisavam ter realizado RM pré-operatória, terem sido submetidos a coleta padronizada de achados intraoperatórios, e ter respondido aos questionários pré e pós-operatório (6, 12 e 24 meses). Não foram incluídos pacientes com rotura associada ou isolada dos tendões do subescapular ou infraespinal, os submetidos a cirurgia aberta, ou aqueles nos quais foi obtido apenas o reparo parcial.

\section{Grupos}

Os pacientes foram divididos em dois grupos: $\mathrm{C}$ e $\mathrm{L}$ ou $\mathrm{U}$, de acordo com Burkhart e Lo. ${ }^{19}$ A categorização foi realizada baseada na inspeção artroscópica. As roturas do tipo $C$ são aquelas que apresentam mobilidade de medial para lateral. As roturas em $\mathrm{L}$ ou em $\mathrm{U}$ apresentam mobilidade principalmente no sentido anteroposterior, e podem necessitar de pontos tendão-tendão ( - Figura $\mathbf{1}$ ).

\section{Desfechos}

A avaliação clínica foi feita pelas escalas The American Shoulder and Elbow Surgeons Standardized Shoulder Assessment (ASES) ${ }^{22,23}$ e Modified-University of California at Los Angeles Shoulder Rating Scale (UCLA). ${ }^{24,25}$

\section{Demais variáveis analisadas}

Variáveis referentes aos pacientes:

- idade, sexo, lado acometido, tabagismo, diabetes.

Variáveis referentes à rotura e à cirurgia:

- padrão da rotura do supraespinal (C versus L ou U);

- retração (pequena, média, grande ou extensa);

- extensão (acometendo a região anterior, posterior ou toda a extensão do tendão);
- grau de degeneração gordurosa da musculatura do manguito rotador (subescapular, supraespinal e infraespinal), de acordo com Goutallier et al.; ${ }^{26}$

- rotura do subescapular (ausente ou parcial);

- número de âncoras utilizadas no reparo;

- realização ou não de acromioplastia;

- realização ou não do procedimento de Mumford;

- procedimento realizado na cabeça longa do bíceps (nenhum, tenotomia ou tenodese);

- reparo com sutura tensa.

Todas as variáveis referentes à lesão, à exceção da degeneração gordurosa, foram analisadas durante a artroscopia. A degeneração gordurosa foi aferida no corte sagital oblíquo T1 de RM de 1,5T.

\section{Análise estatística}

Submetemos as variáveis contínuas à avaliação da normalidade, por meio do teste de Kolmogorov-Smirnov, e da homogeneidade, por meio do teste de Levene. Apresentamos as variáveis contínuas em médias e desvio padrão, e as categóricas, em valores absoluto e percentual.

A comparação entre o padrão da rotura do supraespinal (C versus $\mathrm{L}$ ou $\mathrm{U}$ ) e os resultados funcionais, de acordo com as escalas da ASES e da UCLA, foi realizada pelo teste de MannWhitney. Para as demais variáveis, foi utilizado o teste de Mann-Whitney para as variáveis contínuas, e do Chi-quadrado para as categóricas.

Utilizamos para a análise dos dados o programa Statistical Package for the Social Sciences (SPSS, IBM Corp., Armonk, NY, EUA), versão 21.0, e nível de significância de $5 \%$.

\section{Resultados}

No período estudado, realizamos 341 reparos artroscópicos do manguito rotador. Não foram incluídos 174 casos, por terem sido submetidos a reparo do subescapular e/ou do infraespinal, ou por ter sido possível apenas o reparo parcial. Dessa maneira, analisamos uma amostra de 167 ombros (de 163 pacientes).

As variáveis referentes aos pacientes que demonstraram o padrão em $C$ têm uma menor proporção de pacientes do sexo feminino e menor frequência de pacientes diabéticos (-Tabela 1).

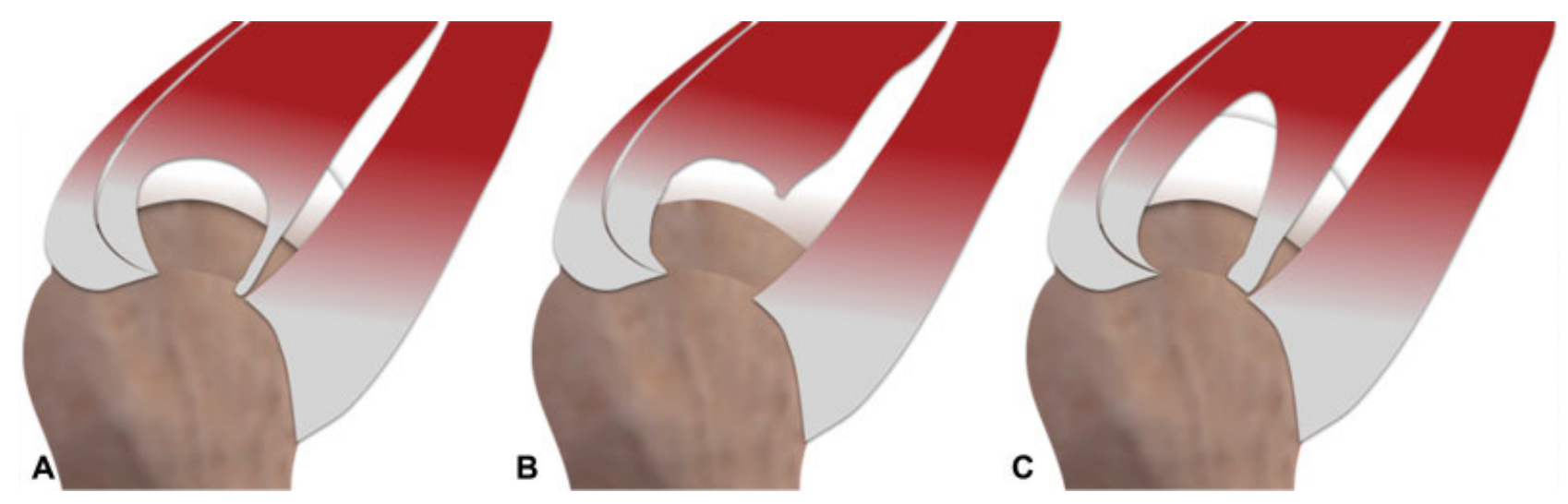

Fig. 1 Padrões de rotura do manguito rotador: (A) lesão em crescente; (B) lesão em L; (C) lesão em U. 
Tabela 1 Características gerais da amostra de acordo com o padrão de rotura do supraespinal

\begin{tabular}{|l|l|l|l|}
\hline & $\begin{array}{l}\text { Rotura do } \\
\text { supraespinal }\end{array}$ & & \\
\hline & $\begin{array}{l}\text { Crescente } \\
\text { (n= 104) }\end{array}$ & $\begin{array}{l}\text { L ou U } \\
\text { (n=63) }\end{array}$ & Valor de $\boldsymbol{p}$ \\
\hline & $\mathbf{n}(\%)$ & $\mathbf{n}(\%)$ & \\
\hline Sexo* & & & \\
\hline Masculino & $44(42)$ & $16(25)$ & $0,027^{*}$ \\
\hline Feminino & $60(58)$ & $47(75)$ & \\
\hline Lado dominante & & & \\
\hline Sim & $71(68)$ & $48(76)$ & 0,273 \\
\hline Não & $33(32)$ & $15(24)$ & \\
\hline Diabetes* & & & \\
\hline Sim & $9(9)$ & $14(22)$ & $0,014^{*}$ \\
\hline Não & $95(91)$ & $49(78)$ & \\
\hline Tabagismo & & & \\
\hline Não & $73(70)$ & $42(67)$ & 0,175 \\
\hline Ex-tabagista & $21(20)$ & $9(14)$ & \\
\hline Tabagista & $10(10)$ & $12(19)$ & \\
\hline $\begin{array}{l}\text { Idade, anos } \\
\text { (média } \pm \text { desvio } \\
\text { padrão) }\end{array}$ & $53,9 \pm 7,9$ & $54,7 \pm 7,9$ & 0,822 \\
\hline
\end{tabular}

$p<0,05$.

As variáveis referentes à cirurgia demonstraram que o padrão em $C$ tem menor retração, menor degeneração gordurosa do supraespinal, e menor necessidade de procedimentos no tendão da cabeça longa do bíceps (-Tabela 2).

No pré-operatório, a escala da ASES demonstrou ser significativamente superior no padrão em $C(43,5 \pm 17,6$ versus $37,7 \pm 13,8 ; p=0,034)$. A escala da UCLA teve 0 mesmo comportamento $(15,2 \pm 4,6$ versus $13,5 \pm 3,6$; $p=0,028)$. No pós-operatório, entretanto, não ocorreu diferença significativa. De acordo com a escala da ASES, o padrão em $C$ foi de $83,7 \pm 18$,7 pontos, e o padrão em $L$ ou $U$, de $82,9 \pm 20,1$ pontos $(p=0,887)$. Respectivamente, os valores foram de $30,9 \pm 4,9$ e de $30,5 \pm 5,6(p=0,773)$ pontos pela escala da UCLA (- Tabela 3 ).

\section{Discussão}

Nosso estudo demonstrou que o padrão da rotura do supraespinal não afetou as escalas funcionais pós-operatórias. O padrão em $C$ teve $83,7 \pm 18,7$ pontos pela escala da ASES, e $30,9 \pm 4,9$ pela escala da UCLA, enquanto o padrão em L ou U teve, respectivamente, $82,9 \pm 20,1$ e $30,5 \pm 5,6$. Esse resultado é consistente com o de outros artigos. ${ }^{20,21}$ Park et al., ${ }^{20}$ estudando roturas grandes, compararam os padrões móveis ( $\mathrm{C}$ e L) com o padrão em U, e também não observaram diferença significativa entre os grupos. Watson et al., ${ }^{21}$ avaliando roturas posterossuperiores, também não observaram diferença entre os grupos. Entretanto, estes autores obser-
Table 2 Características estruturais e procedimentos cirúrgicos de acordo com o padrão de rotura do supraespinal

\begin{tabular}{|c|c|c|c|}
\hline & \multicolumn{3}{|c|}{ Rotura do supraespinal } \\
\hline & $\begin{array}{l}\text { Crescente } \\
(n=104)\end{array}$ & $\begin{array}{l}L \text { ou } U \\
(n=63)\end{array}$ & $\begin{array}{l}\text { Valor } \\
\text { de } p\end{array}$ \\
\hline & n (\%) & n (\%) & \\
\hline \multicolumn{4}{|l|}{ Retração* } \\
\hline Pequena & $64(62)$ & $17(27)$ & $<0,001^{*}$ \\
\hline Média & $37(36)$ & $31(49)$ & \\
\hline Grande & $2(2)$ & $11(17)$ & \\
\hline Extensa & $1(1)$ & $4(6)$ & \\
\hline \multicolumn{4}{|l|}{ Extensão* } \\
\hline $\begin{array}{l}\text { Região anterior } \\
\text { do supraespinal }\end{array}$ & $57(55)$ & $41(65)$ & 0,95 \\
\hline $\begin{array}{l}\text { Região posterior } \\
\text { do supraespinal }\end{array}$ & $28(27)$ & $8(13)$ & \\
\hline Toda a extensão & $19(18)$ & $14(22)$ & \\
\hline \multicolumn{4}{|c|}{$\begin{array}{l}\text { Degeneração gordurosa } \\
\text { supraespinal* }\end{array}$} \\
\hline 0 & $54(52)$ & $18(29)$ & $0,004^{*}$ \\
\hline 1 & $44(42)$ & $32(51)$ & \\
\hline 2 & $6(6)$ & $11(17)$ & \\
\hline 3 & $0(0)$ & $2(3)$ & \\
\hline \multicolumn{4}{|c|}{ Rotura do subescapular } \\
\hline Não & $66(63)$ & $38(60)$ & 0,685 \\
\hline Parcial & $38(37)$ & $25(40)$ & \\
\hline \multicolumn{4}{|l|}{ Número de âncoras } \\
\hline 1 & $33(32)$ & $29(46)$ & 0,179 \\
\hline 2 & $67(64)$ & $32(51)$ & \\
\hline 3 & $4(4)$ & $2(3)$ & \\
\hline \multicolumn{4}{|l|}{ Acromioplastia } \\
\hline Sim & $94(90)$ & $56(89)$ & 0,757 \\
\hline Não & $10(10)$ & $7(11)$ & \\
\hline \multicolumn{4}{|l|}{$\begin{array}{l}\text { Procedimento } \\
\text { de Mumford }\end{array}$} \\
\hline Sim & $6(6)$ & $1(2)$ & 0,191 \\
\hline Não & $98(94)$ & $62(98)$ & \\
\hline \multicolumn{4}{|l|}{$\begin{array}{l}\text { Procedimento } \\
\text { no bíceps* }\end{array}$} \\
\hline Nenhum & $79(76)$ & $33(52)$ & $0,007^{*}$ \\
\hline Tenotomia & $11(11)$ & $12(19)$ & \\
\hline Tenodese & $14(13)$ & $18(29)$ & \\
\hline \multicolumn{4}{|l|}{ Sutura tensa* } \\
\hline Sim & $5(5)$ & $2(3)$ & 0.61 \\
\hline Não & $99(95)$ & $61(97)$ & \\
\hline
\end{tabular}

$p<0,05$.

varam que a melhora obtida em relação ao pré-operatório foi superior no grupo em C, embora não de maneira significativa (34,7 pontos versus 29,5 no grupo em L). 
Table 3 Avaliação funcional pré e pós-operatória de acordo com o padrão da rotura

\begin{tabular}{|l|l|l|l|}
\hline & \multicolumn{2}{|l|}{ Rotura do supraespinal } \\
\hline & $\begin{array}{l}\text { Crescente } \\
(\mathbf{n = 1 0 4 )}\end{array}$ & $\begin{array}{l}\text { L ou U } \\
(\mathbf{n}=\mathbf{6 3})\end{array}$ & Valor de $\boldsymbol{p}$ \\
\hline American Shoulder and Elbow Surgeons Standardized Shoulder Assessment & & & \\
\hline Pré-operatório (média \pm desvio padrão) & $43,5 \pm 17,6$ & $37,7 \pm 13,8$ & 0,034 \\
\hline 24 meses pós (média \pm desvio padrão) & $83,7 \pm 18,7$ & $82,9 \pm 20,1$ & 0,887 \\
\hline Modified-University of California at Los Angeles Shoulder Rating Scale & & & \\
\hline Pré-operatório (média \pm desvio padrão) & $15,2 \pm 4,6$ & $13,5 \pm 3,6$ & 0,028 \\
\hline 24 meses pós (média \pm desvio padrão) & $30,9 \pm 4,9$ & $30,5 \pm 5,6$ & 0,773 \\
\hline
\end{tabular}

Observamos que o padrão em C apresentou valores estatisticamente superiores no pré-operatório de acordo com as escalas da ASES $(43,5 \pm 17,6$ versus $37,7 \pm 13,8 ; p=0,034) \mathrm{e}$ da UCLA (15,2 $\pm 4,6$ versus $13,5 \pm 3,6 ; p=0,028)$. Apesar da diferença mínima clinicamente significante não ter sido alcançada, ${ }^{27}$ este achado difere do dos demais estudos. ${ }^{20,21}$ Semelhantemente a Watson et al., ${ }^{21}$ as lesões em C mostraram menor dimensão, mas diferentemente desses autores, nossa amostra demonstrou número significativamente inferior de mulheres e diabéticos no tipo em C. Observamos ainda maior degeneração gordurosa e maior necessidade de procedimento no bíceps no padrão em L ou U, dado não analisado por esses autores.

A melhora funcional com o procedimento foi maior nas lesões em $\mathrm{L}$ ou $\mathrm{U}$, partindo de um estado funcional pior e atingindo o mesmo nível do que os pacientes com lesões em $C$. Isso ocorreu apesar de as lesões em $\mathrm{L}$ ou $\mathrm{U}$ terem maior retração e maior degeneração gordurosa. Uma possível explicação para isso é o fato de termos avaliado uma amostra predominante de pacientes com degeneração classificada até o grau 2 de Goutallier em ambos os grupos. Apenas 3\% do grupo L ou U foram classificados como grau 3, e não houve pacientes classificados como grau 4 em nenhum dos grupos. Além disso, as roturas se restringiram ao supraespinal. Sabe-se que a degeneração gordurosa, em especial a do infraespinal, gera piores resultados estruturais, ${ }^{9}$ embora o efeito no resultado clínico não seja estatisticamente significante. ${ }^{14,15}$ A dimensão da rotura, por sua vez, é um fator de risco para piores resultados clínicos. ${ }^{14}$ Nossos dados demonstram que o padrão da rotura influenciou no grau de degeneração gordurosa, mas não no resultado funcional pós-operatório.

O cabo dos rotadores é importante na transmissão de força do supraespinal para o úmero, mesmo na presença de uma rotura. ${ }^{19}$ Esta estrutura é normalmente preservada nas roturas em $C$, o que pode explicar a pior função pré-operatória nas lesões em L ou U, e o maior ganho funcional após sua restauração anatômica. Da mesma maneira, consideramos que esse pode ser o motivo do maior grau de degeneração gordurosa pré-operatória nas roturas em L ou U.

Nosso estudo tem algumas limitações. Em primeiro lugar, analisamos apenas o supraespinal, excluindo reparos envolvendo o subescapular e/ou o infraespinal. Essa opção, embora diminua a validade externa, foi escolhida como forma de aumentar a validade interna e reduzir os fatores de confusão. O desenho do tipo coorte retrospectivo, embora similar ao de estudos prévios, ${ }^{20,21}$ também é uma possível fonte de viés. A análise intraoperatória por apenas um cirurgião acrescenta subjetividade à classificação. Por fim, também não realizamos a análise estrutural do reparo, diferentemente de Park et al. ${ }^{20}$ Entretanto, sabe-se que a integridade estrutural não se correlaciona de maneira clinicamente significativa com os resultados funcionais após o reparo do manguito rotador, ${ }^{28}$ e a análise clínica isoladamente já foi realizada por outros autores. ${ }^{21}$

Como pontos favoráveis destacamos a análise padronizada de roturas do supraespinal numa grande casuística, superior à dos estudos prévios, ${ }^{20,21}$ e a demonstração de que, embora não influencie nos resultados pós-operatórios, o padrão da rotura pode influenciar na avaliação préoperatória.

\section{Conclusões}

As roturas do supraespinal em C e em L ou U apresentam resultados funcionais pós-operatórios semelhantes. No préoperatório, as roturas em C apresentam função estatisticamente superior.

Conflito de interesses

Os autores declaram não haver conflito de interesses.

\section{Referências}

1 Yamamoto A, Takagishi K, Osawa T, et al. Prevalence and risk factors of a rotator cuff tear in the general population. J Shoulder Elbow Surg 2010;19(01):116-120

2 Checchia SL, Santos PD, Miyazaki AN, et al. Avaliação dos resultados obtidos na reparação artroscópica das lesões do manguito rotador. Rev Bras Ortop 2005;40(05):229-238

3 Godinho GG, França FO, Freitas JMA, et al. Avaliação da integridade anatômica por exame de ultrassom e funcional pelo índice de Constant \& Murley do manguito rotador após reparo artroscópico. Rev Bras Ortop 2010;45(02):174-180

4 Veado MA, Almeida Filho IA, Duarte RG, Leitão I. Avaliação funcional do reparo artroscópico das lesões completas do manguito rotador associado a acromioplastia. Rev Bras Ortop 2008;43 (11-12):505-512

5 McElvany MD, McGoldrick E, Gee AO, Neradilek MB, Matsen FA III. Rotator cuff repair: published evidence on factors associated with repair integrity and clinical outcome. Am J Sports Med 2015;43 (02):491-500 
6 Shin YK, Ryu KN, Park JS, Jin W, Park SY, Yoon YC. Predictive Factors of Retear in Patients With Repaired Rotator Cuff Tear on Shoulder MRI. AJR Am J Roentgenol 2018;210(01):134-141

7 Rashid MS, Cooper C, Cook J, et al. Increasing age and tear size reduce rotator cuff repair healing rate at 1 year. Acta Orthop 2017; 88(06):606-611

8 Kim YK, Jung KH, Kim JW, Kim US, Hwang DH. Factors affecting rotator cuff integrity after arthroscopic repair for medium-sized or larger cuff tears: a retrospective cohort study. J Shoulder Elbow Surg 2018;27(06):1012-1020

9 Park JS, Park HJ, Kim SH, Oh JH. Prognostic Factors Affecting Rotator Cuff Healing After Arthroscopic Repair in Small to Medium-sized Tears. Am J Sports Med 2015;43(10):2386-2392

10 Nho SJ, Brown BS, Lyman S, Adler RS, Altchek DW, MacGillivray JD. Prospective analysis of arthroscopic rotator cuff repair: prognostic factors affecting clinical and ultrasound outcome. JShoulder Elbow Surg 2009;18(01):13-20

11 Le BTN, Wu XL, Lam PH, Murrell GAC. Factors predicting rotator cuff retears: an analysis of 1000 consecutive rotator cuff repairs. Am J Sports Med 2014;42(05):1134-1142

12 Jeong HY, Kim HJ, Jeon YS, Rhee YG. Factors Predictive of Healing in Large Rotator Cuff Tears: Is It Possible to Predict Retear Preoperatively? Am J Sports Med 2018;46(07):1693-1700

13 Gulotta LV, Nho SJ, Dodson CC, Adler RS, Altchek DW, MacGillivray JD; HSS Arthroscopic Rotator Cuff Registry. Prospective evaluation of arthroscopic rotator cuff repairs at 5 years: part II-prognostic factors for clinical and radiographic outcomes. JShoulder Elbow Surg 2011;20(06):941-946

14 Pécora JO, Malavolta EA, Assunção JH, Gracitelli MEC, Martins JPS, Ferreira AA Jr. Prognostic factors for clinical outcomes after rotator cuff repair. Acta Ortop Bras 2015;23(03):146-149

$15 \mathrm{Oh} \mathrm{JH}$, Kim SH, Ji HM, Jo KH, Bin SW, Gong HS. Prognostic factors affecting anatomic outcome of rotator cuff repair and correlation with functional outcome. Arthroscopy 2009;25(01):30-39

16 Millett PJ, Espinoza C, Horan MP, et al. Predictors of outcomes after arthroscopic transosseous equivalent rotator cuff repair in 155 cases: a propensity score weighted analysis of knotted and knotless self-reinforcing repair techniques at a minimum of 2 years. Arch Orthop Trauma Surg 2017;137(10):1399-1408

17 Jenssen KK, Lundgreen K, Madsen JE, Kvakestad R, Dimmen S. Prognostic Factors for Functional Outcome After Rotator Cuff
Repair: A Prospective Cohort Study With 2-Year Follow-up. Am J Sports Med 2018;46(14):3463-3470

18 Fermont AJ, Wolterbeek N, Wessel RN, Baeyens JP, de Bie RA. Prognostic factors for recovery after arthroscopic rotator cuff repair: a prognostic study. J Shoulder Elbow Surg 2015;24(08):1249-1256

19 Burkhart SS, Lo IKY. Arthroscopic rotator cuff repair. JAm Acad Orthop Surg 2006;14(06):333-346

20 Park JY, Jung SW, Jeon SH, Cho HW, Choi JH, Oh KS. Arthroscopic repair of large U-shaped rotator cuff tears without margin convergence versus repair of crescent- or L-shaped tears. Am J Sports Med 2014;42(01):103-111

21 Watson S, Allen B, Robbins C, Bedi A, Gagnier JJ, Miller B. Does the Rotator Cuff Tear Pattern Influence Clinical Outcomes After Surgical Repair? Orthop J Sports Med 2018;6(03):2325967118763107

22 Richards RR, An KN, Bigliani LU, et al. A standardized method for the assessment of shoulder function. J Shoulder Elbow Surg 1994; 3(06):347-352

23 Knaut LA, Moser ADL, Melo SdeA, Richards RR. Translation and cultural adaptation to the portuguese language of the American Shoulder and Elbow Surgeons Standardized Shoulder assessment form (ASES) for evaluation of shoulder function. Rev Bras Reumatol 2010;50(02):176-189

24 Ellman H, Hanker G, Bayer M. Repair of the rotator cuff. End-result study of factors influencing reconstruction. J Bone Joint Surg Am 1986;68(08):1136-1144

25 Oku EC, Andrade AP, Stadiniky SP, Carrera EF. Tradução e adaptação cultural do Modified-University of California at Los Angeles Shoulder Rating Scale para a língua portuguesa. Rev Bras Reumatol 2006;46(04):246-252

26 Goutallier D, Postel JM, Bernageau J, Lavau L, Voisin MC. Fatty muscle degeneration in cuff ruptures. Pre- and postoperative evaluation by CT scan. Clin Orthop Relat Res 1994;(304):78-83

27 Gagnier JJ, Robbins C, Bedi A, Carpenter JE, Miller BS. Establishing minimally important differences for the American Shoulder and Elbow Surgeons score and the Western Ontario Rotator Cuff Index in patients with full-thickness rotator cuff tears. J Shoulder Elbow Surg 2018;27(05):e160-e166

28 Russell RD, Knight JR, Mulligan E, Khazzam MS. Structural integrity after rotator cuff repair does not correlate with patient function and pain: a meta-analysis. J Bone Joint Surg Am 2014;96 (04):265-271 